\title{
Risk factors for Accidental Foreign Body Aspiration and Ingestion in Dental Practice: A Seven-Year Retrospective Study
}

Jisun Huh

Yonsei University College of Dentistry https://orcid.org/0000-0001-5514-187X

Ki-Yeol Kim

Yonsei University College of Dentistry

Seoyeon Jung

Yonsei University College of Dentistry

Jungyul Cha

Yonsei University College of Dentistry

Kee-Deog Kim

Yonsei University College of Dentistry

Wonse Park ( $\nabla$ wonse@yuhs.ac)

https://orcid.org/0000-0002-2081-1156

Research article

Keywords: accidental foreign body aspiration and ingestion, incidence rate, dental treatment

Posted Date: April 8th, 2020

DOl: https://doi.org/10.21203/rs.3.rs-21596/v1

License: (9) (i) This work is licensed under a Creative Commons Attribution 4.0 International License.

Read Full License 


\section{Abstract}

Objective: This retrospective study investigated the incidence rate of accidental foreign body aspiration and ingestion according to patient sex and age and the dental department. This study was aimed to verify the risk factors for accidental foreign body aspiration and ingestion in dental practice.

Methods: Accidental foreign body aspiration and ingestion cases were collected by searching the electronic health records and the Safety Report System of Yonsei University Dental Hospital from January 2011 to December 2017. The collected data comprised the patient age, sex, and medical condition, treatment procedure, and foreign objects that were accidentally aspirated or ingested. The incidence rate was calculated as the number of accidental foreign body aspirations and ingestions relative to the total number of patient visits, and differences according to the patient sex and age and the dental department were identified statistically.

Results: There were 2 aspiration and 37 ingestion cases during the 7-year analysis period. The male-tofemale incidence ratio was 22.7:8.1. The incidence rate trended upward with age and increased rapidly among those aged 80 or older. Seven of the 39 patients had intellectual disability, Lou Gehrig's disease, dystonia, or oral and maxillofacial cancer. The incidence rate was the highest in the Predoctoral Student Clinic and the Department of Prosthodontics. The most frequently swallowed objects were fixed dental prostheses and dental implant components.

Conclusion: The incidence rate of accidental foreign body aspiration and ingestion is higher in men than in women and the highest in patients aged 80 or older. The most frequently aspirated or ingested objects are fixed dental prostheses and dental implant components. Dental practitioners need to identify high-risk patients and apply various methods to prevent accidental foreign body aspiration and ingestion in dental clinics. Inexperienced dentists should be especially careful. Keywords: accidental foreign body aspiration and ingestion, incidence rate, dental treatment

\section{Background}

Accidental foreign body aspiration and ingestion is an emergency situation that often occurs in dental clinics. Many objects can potentially be aspirated or ingested, including endodontic instruments, orthodontic materials, and dental implant components[1-3]. Ingestion occurs more frequently than aspiration, and the frequency is influenced by the patient age and medical condition, and the type of dental procedure[4-6].

Most of the articles on accidental foreign body aspiration and ingestion in dental clinics in the literature are case reports. Even in review articles, it is difficult to obtain systematic information on the incidence rate of accidental foreign body aspiration and ingestion and the influencing factors due to data inconsistency between different articles. 
This retrospective study analyzed accidental foreign body aspiration and ingestion cases that occurred at Yonsei University Dental Hospital over a 7-year period. The incidence rate of accidental foreign body aspiration and ingestion and the affecting factors such as the patient age, sex, and medical condition, and the dental treatment involved were analyzed. We identified what information should be included in predoctoral and continuing education to prevent accidental foreign body aspiration and ingestion based on an analysis of the present cases.

\section{Methods}

We collected and analyzed data as follows to find out the incidence rate of accidental foreign body aspiration and ingestion according to sex, age, and dental department.

\section{Data collection and analysis}

We searched electronic health records (EHRs) for patients who visited Yonsei University Dental Hospital from January 2011 to December 2017. All of the following patient inclusion criteria needed to be satisfied:

1)

Visited a medical department on the day of the dental visit or the following day.

2)

Underwent neck, chest, or abdominal radiography, laryngoscopy, or endoscopy on that day.

3)

The presence of a foreign body stated in the EHR.

4)

The occurrence of accidental foreign body aspiration and ingestion confirmed in the EHR.

We additionally included accidental foreign body aspiration and ingestion cases reported in the Safety Report System of Yonsei University Dental Hospital from January 2011 to December 2017.

The collected data were the patient age, sex, and medical condition, the dental department where accidental foreign body aspiration or ingestion occurred, the dental treatment applied, the aspirated or ingested foreign body, and the medical examination, treatment, and progress.

During the same period, the total number of patient visits by sex, age, and clinical department were reviewed, and the incidence rate was calculated as the ratio of the number of cases of accidental foreign body aspiration and ingestion relative to the total number of patient visits. The incidence rates according to patient age and sex and the dental department were calculated.

\section{Statistical analysis}

The incidence rates according to sex, age, and clinical department were compared using Chi-square test. The data analysis was performed using SPSS ver. 25.0 software (SPSS Inc., Chicago, IL), with a 
significance criterion of $p<0.01$.

\section{Results}

\section{Incidence}

There were 39 cases of foreign body aspiration $(n=2)$ and ingestion $(n=37)$ identified during the 7-year study period. The 37 ingestion cases included 7 patients with the following conditions that could affect swallowing or cognition: 1 with intellectual disability, 1 with Lou Gehrig's disease, 1 with oromandibular dystonia, and 4 with a history of extensive resection due to oral or maxillofacial cancer (3 with oral cancer and 1 with sinus cancer).

\section{Medical progress}

One of the two aspiration cases was an 83-year-old female who aspirated the crown portion of a resected tooth during extraction. She exhibited no major symptom such as shortness of breath. After a failed attempt to remove the object by bronchoscopy on the same day, it was removed using rigid bronchoscopy under general anesthesia on the following day. The other case was a 77-year-old male who aspirated a fixed dental prosthesis that was removed for extraction. The aspirated object was removed by bronchoscopy under conscious sedation.

The 37 ingestion cases comprised 16 ingested foreign bodies removed by endoscopy and 21 cases that were followed up. There were no medical complications such as abdominal pain or gastrointestinal bleeding, with only one case of a minor mucosal scratch that occurred while removing an ingested highspeed bur.

\section{Aspirated and ingested objects (Fig. 1)}

Crowns and bridges were the objects that were most frequently aspirated or ingested during try-in, cementation, or the removal of prostheses for extraction. The second most common objects were dental implant components. Three cases of swallowing low-speed contra-angle dental burs during composite polishing occurred in the Predoctoral Student Clinic. The other cases included the lip clip of an electronic apex locator during endodontic treatment, a suture needle during suturing after extraction, and the fractured fragment of an ultrasonic scaler tip during scaling.

\section{Incidence rate by sex (Fig. 2)}

The 39 cases occurred in 12 females ( 1 aspiration and 11 ingestion) and 27 males ( 1 aspiration and 26 ingestion). The incidence rate was 22.7 per million for males and 8.1 per million for females, with a significant sex difference $(p=0.002)$.

\section{Incidence rate by age (Fig. 3)}

The incidence rate ranged from 2.7 to 14.4 per million among patients younger than 60 years, and increased to 22.2 and 32.6 per million among those aged 60-69 and 70-79 years, respectively, and 
markedly to 100.5 per million for those aged 80 or older. The incidence rate differed significantly between the age groups $(p<0.001)$.

\section{Incidence rate by department (Fig. 4)}

The incidence rate was highest in the Predoctoral Student Clinic, at 66.7 per million, followed by (in decreasing order) the Department of Prosthodontics (44.6 per million), Department of Advanced General Dentistry (20.4 per million), Department of Conservative Dentistry (15.0 per million), Department of Oral and Maxillofacial Surgery (14.8 per million), Department of Pediatric Dentistry (5.9 per million), and Department of Periodontics (5.5 per million). No cases of accidental foreign body aspiration and ingestion occurred in the Department of Orthodontics and Oral Medicine. The incidence rate differed significantly between departments $(p<0.001)$.

\section{Discussion}

This study identified 2 cases of accidental foreign body aspiration and 37 cases of accidental foreign body ingestion that occurred over a 7-year period at a single dental hospital. Severe complications did not occur, and no surgical intervention was needed. The incidence rate was higher in males than in females, and increased markedly in old patients aged 80 or older. It was highest in the Predoctoral Student Clinic and second highest in the Department of Prosthodontics. The most frequently swallowed foreign bodies were fixed dental prostheses and dental implant components.

A particularly interesting finding of this study was the incidence rate being more than twofold higher in males than in females. Another 7-year-long retrospective study analyzing cases of the endoscopic removal of dental foreign bodies in a medical and dental hospital in Japan found a male-to-female ratio of 21:8[7], which is similar to that in the present study. The anatomies of the larynx and pharynx are known to differ between males and females, with the oropharynx volume being larger in males[8]. In addition to anatomical differences, tongue strength and endurance might also affect the incidence rate, although sex-related differences in these parameters are controversial $[9,10]$.

The higher incidence of accidental foreign body aspiration and ingestion in older people is consistent with the findings of previous studies, which may be explained by the effects of a decreased gag reflex and an increased prevalence of various medical conditions[5]. Foreign body aspiration and ingestion was reported to occur frequently in children and rarely in adults[11,12], which contrasts to the present study finding a low incidence in children. This might be due to various methods for preventing accidental foreign body aspiration and ingestion being applied more actively for children. In the Department of Pediatric Dentistry of Yonsei University Dental Hospital, rubber dams are strictly applied during all possible treatments, with other preventive measures such as gauze throat pack being used when performing a dental procedure in which it is impossible to use a rubber dam.

It is noteworthy that 7 of the 39 patients in this study had impaired cognition or conditions that may have affected swallowing. Patients who receive excisional surgeries for oral and maxillofacial cancer and 
those with oral and maxillofacial deformities may be at a higher risk of accidental foreign body aspiration and ingestion, due to their throat being open during dental treatment. There have been several case reports of accidental foreign body aspiration and ingestion in patients with Parkinson's disease, stroke, and psychiatric problems[13] [14]. The present retrospective study has confirmed that these patients are at a high risk.

The high incidence rate found in the Predoctoral Student Clinic in this study is consistent with other studies finding that accidental foreign body ingestion occurs more frequently when procedures are performed by unskilled practitioners $[15,16]$. This is consistent with unskilled practitioners probably being more likely to make various mistakes compared to skilled operators. We found three cases of accidental ingestion of low-speed contra-angle dental burs in the Predoctoral Student Clinic, which were due to obvious bur-fastening errors-experienced practitioners rarely make such mistakes.

If object loss is identified in the oral cavity and the object is not found in the treatment field, the presence of the foreign body within the respiratory or digestive tract should be confirmed using radiography, laryngoscopy, or bronchoscopy. There might be no symptoms if the foreign body is aspirated[17]. Urgent endoscopic intervention may be required depending on the type and location of the foreign body in cases of ingestion. Ingested foreign bodies that are sharp are much more likely to cause complications, and so they must be removed[11]. A case report found that a dental implant screwdriver that was swallowed had remained in the intestine for more than 7 days, and so colonoscopy under general anesthesia was needed to remove it. Ingested foreign bodies for which the decision is made not to remove them must be followed up until they are excreted.

It is crucial to apply interventions aimed at preventing the aspiration and ingestion of foreign bodies. First of all, dental practitioners need to identify high-risk patients. Patients aged 80 or older can be considered to be at a very high risk. Patients with conditions that cause muscle weakness, decreased reflexes, cognitive impairment, and which can affect swallowing are also at a high risk. All possible preventive measures should be actively applied for such high-risk patients.

A rubber dam is the most obvious preventive measure, and one that completely covers the oral cavity should be used whenever possible. Alternatives may be used in situations where a rubber dam is not suitable. It is advisable to wear tight gloves and not to recline the chair too much (i.e., only to the semisupine position) when handling small objects within the oral cavity or when extracting deciduous teeth. It might be prudent to use floss to secure objects such as rubber dam clamps, bridge restorations, dental implant screwdrivers, and endodontic files. Placing a gauze throat pack is also recommended. However, since such gauze can be swallowed, the use of $4 \times 4$ gauze with a radiopaque thread that can be seen in case of swallowing is recommended over the $2 \times 2$ size.

Checking that dental burs are properly installed is useful for preventing them from dropping into the oral cavity. When a dental bur is mounted on a low-speed contra-angle handpiece, it should be pushed all the way in and then pulled back in order to check that it does not come out. Activating a handpiece before using it within oral cavity will check that the bur is installed properly and does not detach from the 
handpiece. Most dental burs are sharp and dangerous objects that can puncture the digestive tract when they are ingested.

The case of a fractured scaler tip fragment being swallowed indicates that dental practitioners should be aware that instruments with a thin tip may fracture. Care should therefore be taken to ensure that instruments do not fracture within the oral cavity by ensuring the use of the correct instrumentation and avoiding applying excessive forces. Dentists should check the connections of instruments with detachable parts, such as dental mirrors. The use of a swaged needle is recommended in order to prevent needle ingestion, given that one case was found in this study.

\section{Conclusion}

The incidence rate of accidental foreign body aspiration and ingestion was higher in male than in female and prominently high in patients aged 80 or older. It was highest in the Predoctoral Student Clinic and the most frequently aspirated of ingested objects were fixed dental prostheses and dental implant components. Dental practitioners need to identify high-risk patients and situations and applying various methods for preventing foreign body aspiration and ingestion. Inexperienced dentists should understand the actual cases and be more careful.

\section{Declarations}

Ethics approval and consent to participate

This retrospective chart review study involving human participants was in accordance with the ethical standards of the institutional and national research committee and with the 1964 Helsinki Declaration and its later amendments or comparable ethical standards. The Institutional Review Board at Yonsei University Dental Hospital approved this study (approval number 2-2019-0074). For this type of study, formal consent is not required.

Consent for publication

Not applicable

Availability of data and materials

The datasets used and/or analyzed during the current study are available from the corresponding author on reasonable request.

Competing interests:

The authors declare that they have no competing interests.

Funding

This work was not supported by any grant.

Authors' contribution

Jisun Huh, Jungyul Cha, Kee-Deog Kim, Wonse Park; Study concept and design: Jisun Huh, Seoyeon Jung; Analysis of data: Jisun Huh, Ki-Yeol Kim, Seoyeon Jung, Wonse Park; Interpretation of data: Ki-Yeol 
Kim; Statistical analysis: Jisun Huh, Ki-Yeol Kim, Seoyeon Jung, Jungyul Cha, Kee-Deog Kim, Wonse Park; Preparation of manuscript, read and approval of the final manuscript

\section{References}

1. Abusamaan M, Giannobile WV, Jhawar P, Gunaratnam NT. Swallowed and aspirated dental prostheses and instruments in clinical dental practice: a report of five cases and a proposed management algorithm. The Journal of the American Dental Association. 2014;145(5):459-63.

2. Milton T, Hearing S, Ireland A. Ingested foreign bodies associated with orthodontic treatment: report of three cases and review of ingestion/aspiration incident management. British dental journal. 2001;190(11):592-6.

3. Susini G, Pommel L, Camps J. Accidental ingestion and aspiration of root canal instruments and other dental foreign bodies in a French population. International endodontic journal. 2007;40(8):585-9.

4. Tiwana KK, Morton T, Tiwana PS. Aspiration and ingestion in dental practice: a 10-year institutional review. The Journal of the American Dental Association. 2004;135(9):1287-91.

5. Cameron SM, Whitlock WL, Tabor MS. Foreign body aspiration in dentistry: a review. J Am Dent Assoc. 1996;127(8):1224-9.

6. Cossellu G, Farronato G, Carrassi A, Angiero F. Accidental aspiration of foreign bodies in dental practice: clinical management and prevention. Gerodontology. 2015;32(3):229-33.

7. Mizuno K-i, Takahashi K, Tominaga K, Nishigaki Y, Sato H, Ikarashi S, Hayashi K, Yamamoto T, Honda Y, Hashimoto S: Endoscopic removal of ingested dentures and dental instruments: a retrospective analysis. Gastroenterology research and practice 2016, 2016.

8. Inamoto Y, Saitoh E, Okada S, Kagaya H, Shibata S, Baba M, Onogi K, Hashimoto S, Katada K, Wattanapan P. Anatomy of the larynx and pharynx: effects of age, gender and height revealed by multidetector computed tomography. J Rehabil. 2015;42(9):670-7.

9. Youmans SR, Youmans GL, Stierwalt JA. Differences in tongue strength across age and gender: is there a diminished strength reserve? Dysphagia 2009, 24(1):57-65.

10. Vanderwegen J, Guns C, Van Nuffelen G, Elen R, De Bodt M. The influence of age, sex, bulb position, visual feedback, and the order of testing on maximum anterior and posterior tongue strength and endurance in healthy Belgian adults. Dysphagia. 2013;28(2):159-66.

11. Eisen GM, Baron TH, Dominitz JA, Faigel DO, Goldstein JL, Johanson JF, Mallery JS, Raddawi HM. Vargo II JJ, Waring JP: Guideline for the management of ingested foreign bodies. Gastrointest Endosc. 2002;55(7):802-6.

12. Boyd M, Chatterjee A, Chiles C, Chin R. Tracheobronchial foreign body aspiration in adults. Southern medical journal. 2009;102(2):171.

13. Deliberador TM, Marengo G, Scaratti R, Giovanini AF, Zielak JC, Baratto Filho F. Accidental aspiration in a patient with Parkinson's disease during implant-supported prosthesis construction: a case report. 
Spec Care Dentist. 2011;31(5):156-61.

14. Worthington P. Ingested foreign body associated with oral implant treatment: report of a case. International Journal of Oral \& Maxillofacial Implants 1996, 11(5).

15. Hisanaga R, Hagita K, Nojima K, Katakura A, Morinaga K, Ichinohe T, Konomi R, Takahashi T, Takano $\mathrm{N}$, Inoue T. Survey of accidental ingestion and aspiration at Tokyo Dental College Chiba Hospital. Bull Tokyo Dent Coll. 2010;51(2):95-101.

16. Obinata K, Satoh T, Towfik AM, Nakamura M. An investigation of accidental ingestion during dental procedures. Journal of oral science. 2011;53(4):495-500.

17. Foltran F, Ballali S, Passali FM, Kern E, Morra B, Passali GC, Berchialla P, Lauriello M, Gregori D. Foreign bodies in the airways: a meta-analysis of published papers. International journal of pediatric otorhinolaryngology. 2012;76:12-9.

\section{Figures}

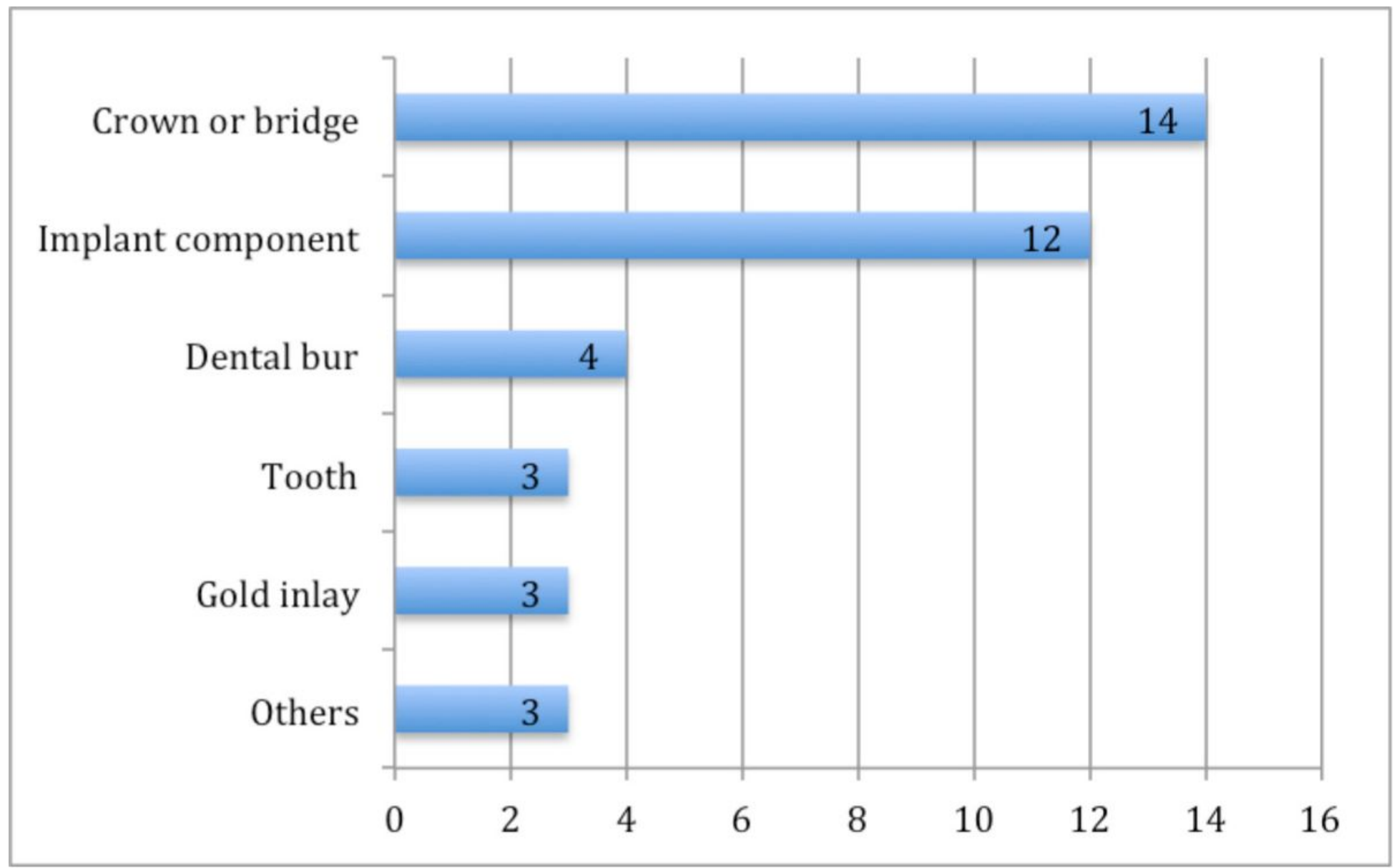

\section{Figure 1}

Aspirated and ingested objects. The most frequently aspirated or ingested foreign bodies were fixed dental prostheses and dental implant components. 


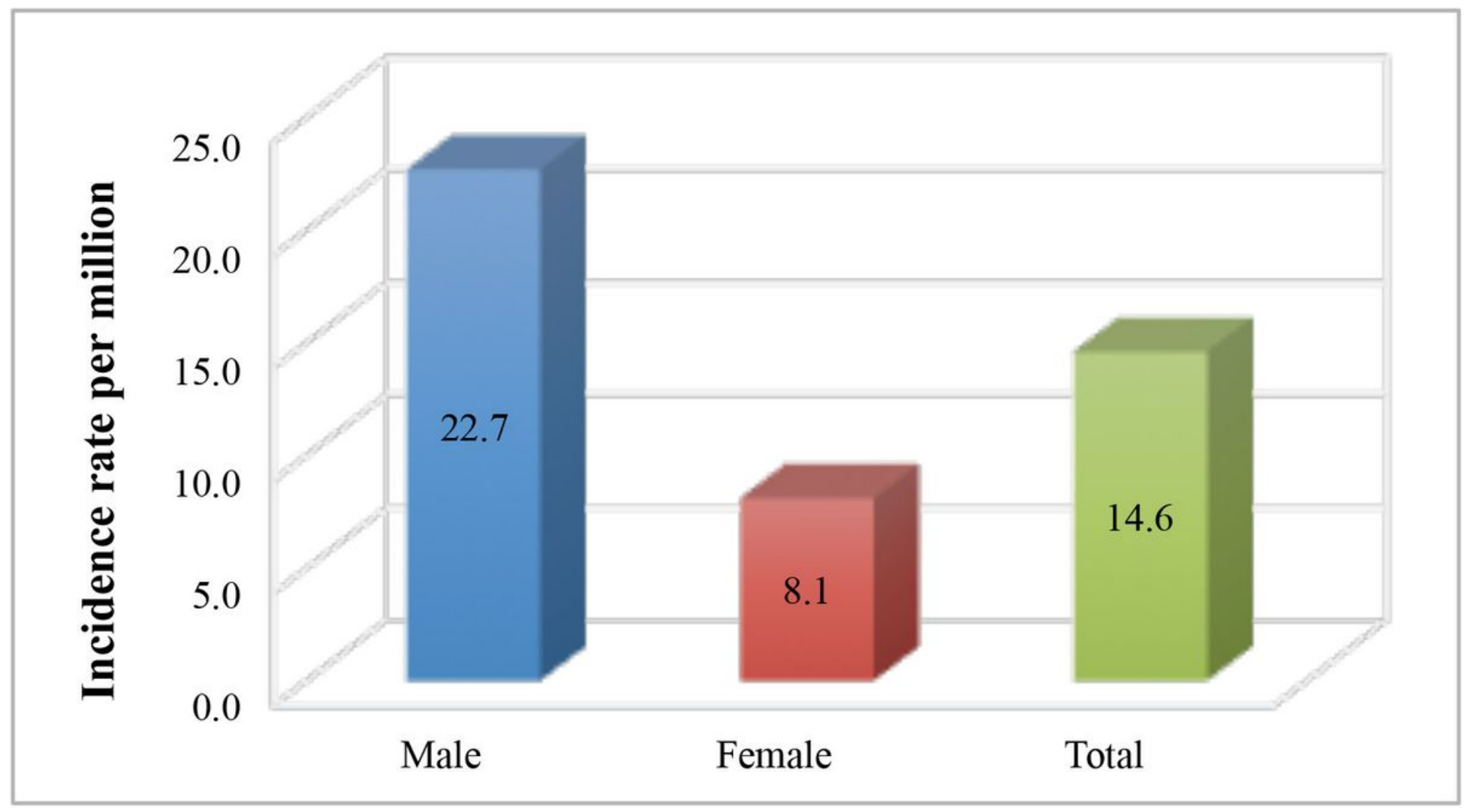

Figure 2

Incidence rate of accidental foreign body aspiration and ingestion by sex. Incidence rate is higher in male than in female, with a significant difference $(p=0.002)$. 


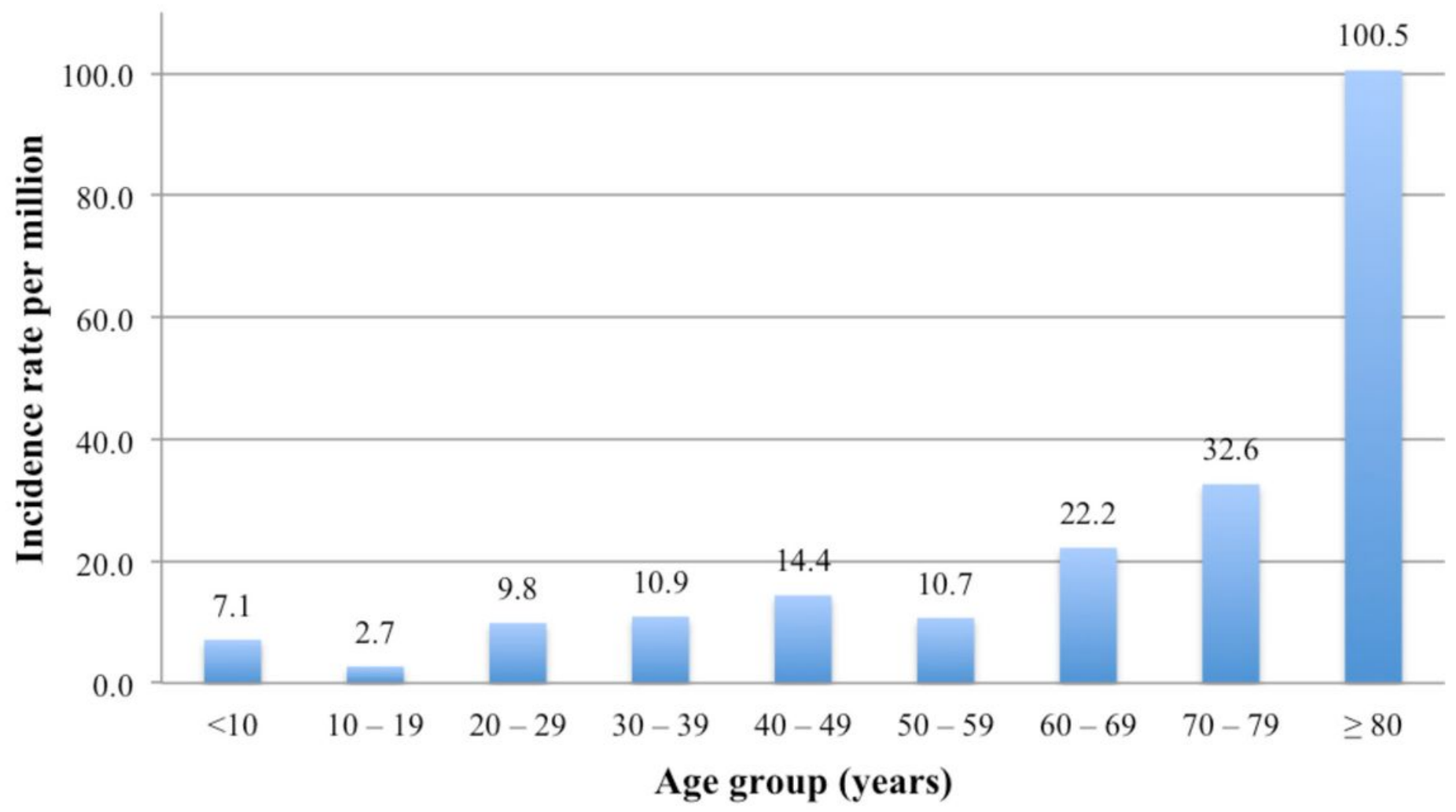

\section{Figure 3}

Incidence rate of accidental foreign body aspiration and ingestion by age group. The incidence rate differed significantly between the age groups $(p<0.001)$. The incidence trended upward with age, with a particularly marked increased in patients aged 80 or older.

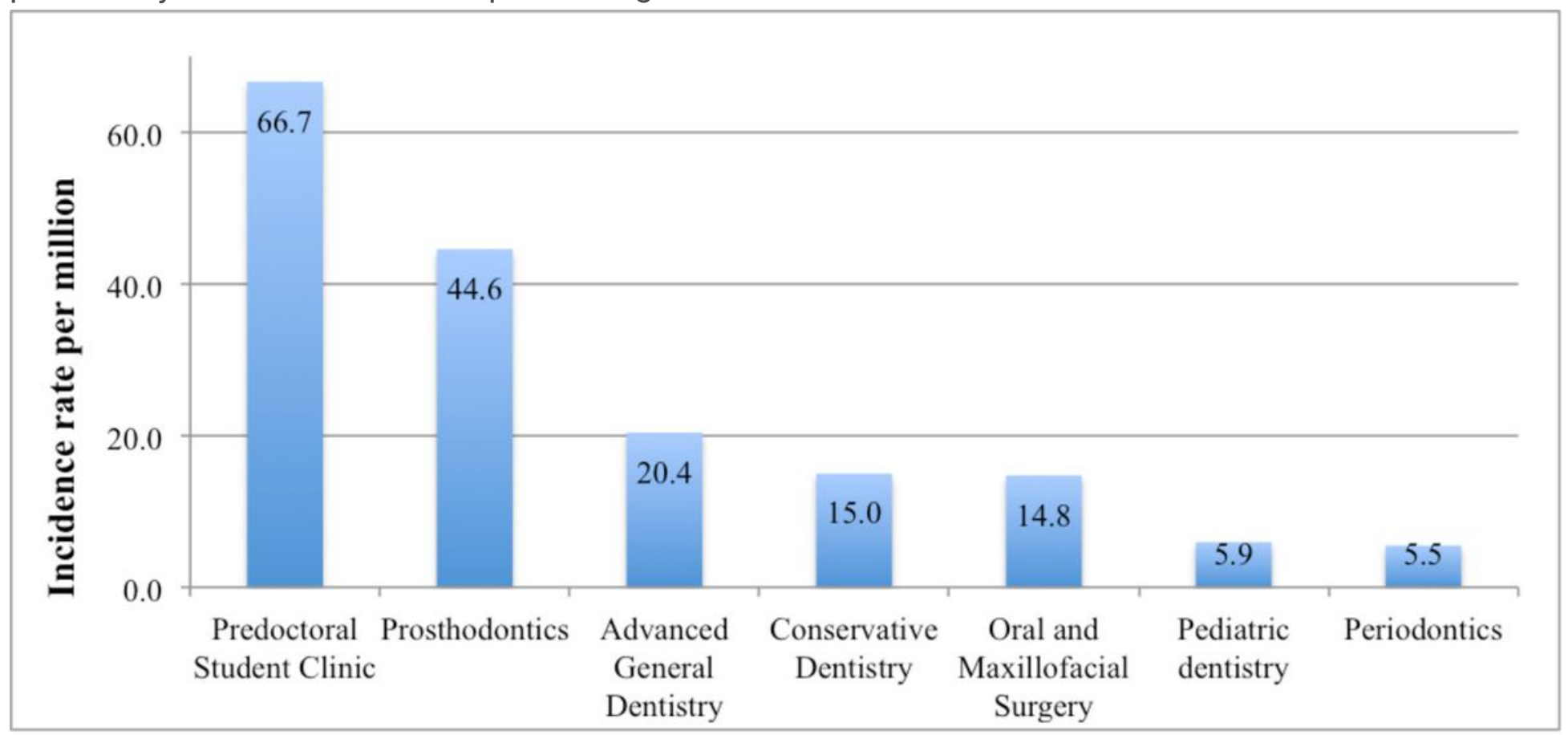




\section{Figure 4}

Incidence rate of accidental foreign body aspiration and ingestion by department. The incident rate differed significantly between departments $(p<0.001)$, and was highest in the Predoctoral Student Clinic, followed by the Department of Prosthodontics. 